\title{
The Design and Implementation of E-Commerce Management System.
}

\author{
SHARMINAKTER \\ M.Sc on Applied Mathematics, University of Dhaka, Bangladesh \\ M.Phil (Enrolled), University of Dhaka, Bangladesh \\ Lecturer; Atish Dipankar University of Science \& Technology (ADUST), Dhaka, Bangladesh, \\ E-mail: sharmindu2005@gmail.com
}

\begin{abstract}
Electronic Commerce is process of doing business through computer networks. A person sitting on his chair in front of a computer can access all the facilities of the Internet to buy or sell the products. Unlike traditional commerce that is carried out physically with effort of a person to go \& get products, ecommerce has made it easier for human to reduce physical work and to save time. E-Commerce which was started in early 1990's has taken a great leap in the world of computers, but the fact that has hindered the growth of ecommerce is security. Security is the challenge facing e-commerce today \& there is still a lot of advancement made in the field of security.The main advantage of e-commerce over traditional commerce is the user can browse online shops, compare prices and order merchandise sitting at home on their PC.For increasing the use of e-commerce in developing countries the B2B e-commerce is implemented for improving access to global markets for firms in developing countries. For a developing country advancement in the field of e-commerce is essential. The research strategy shows the importance of the e-commerce in developing countries for business applications.
\end{abstract}

Keywords: Product display tools, Shopping cart, Payment system, Business to consumer (B2C), Business to Business (B2B), Business to Government (B2G), Business to Consumer (B2C), Consumer to Business (C2B) etc.

\section{Introduction}

As the retail industry continues to change rapidly, retailers need increasingly sophisticated ecommerce management solutions to stay competitive. Consumers today expect to engage in Omni channel ecommerce, using multiple devices and moving between online and offline channels with ease. Unaware of the complications this creates for retailers, consumers expect merchants to seamlessly accommodate their Omni channel shopping experience. But only the most advanced ecommerce management technology can deliver the infrastructure to meet consumer demands today and to integrate the new ecommerce technologies that will emerge tomorrow.We are going to highlight various constraints to commerce and trade in general and ecommerce in particular. Many of these constraints are endemic across the business sector and demands major reforms through strong political commitment and an ability to implement policy changes. A list of specific constraints to e-commerce that policy makers can address in the short and medium term is summarized below.

- Too few telephone connections.

- Absence of a strong independent regulatory body for the telecommunication sector.

- Absence of encryption law that precludes acceptance of digital signature.

- Strong dependence of Letter of Credit to conduct international transactions.

- Non-issuance of international credit cards for cross border transactions.

- Interest rate ceiling on export loans.

Now these days online order through shopping cart has made the life very straightforward for both customers and shoppers. The customer can browse different items by sitting in their home with comfort. But this is on the form of $\mathrm{C} 2 \mathrm{C}$ (Consumer to Consumer) e-commerce area. The form of B2B (Business to Business) is getting very popular also and making the business very easy, trouble free era. We want construct a unique B2C solution e-commerce portal. Through which buyers of every region of the word will be able to get our every their products in one platform. This portal will be work as an online directory of Bangladesh.

\section{Proposed Model}

The commonly listed functions and features revolve mainly around the transaction processing capabilities of an e-commerce system. As noted earlier, however, e-commerce systems, like most information systems, provide information to support the management of the organization. It brings into being a virtual marketplace with new relationships among businesses and consumers.E-commerce represents not only the ability to purchase and sell goods and services via a digital medium such as the internet, but also the ability to 
automate the entire sales and purchase process. A typical goods or services business will have the following business processes:

- Customer support

- Sales

- Refunds

- Inventory management

- Customer information management

- Marketing

E-commerce can be divided into primarily three categories:

$>$ Business to consumer (B2C): Where enterprises sell directly to the customer, often cutting out ('disinter mediating') wholesalers or 'bricks and mortars' retail outlets. B2C is the most commonly understood form of internet business (www.amazon.com). The most successful trading has been with standard products such as CDs, Books, Software, downloadable music etc.

$>$ Business to Business (B2B): it is larger, growing faster. This includes procurements of raw materials and supplies, liaison with contractors, sales channels, servicing customers, collaborating with partners, integrated management with data and knowledge.

> Business to Government (B2G): Here business trade directly with government offices and agencies for public procurement (e.g. supplies for hospitals, school and other government contracts.

$>$ Consumer to consumer (C2C): Online transaction between private individuals.

$>$ Consumer to business $(\mathrm{C} 2 \mathrm{~B})$

\subsection{Components Of An E-Commerce System}

An E-commerce system consists of a number of vital components:

- A database

- E-commerce website

- Security system

- Payment gateway

These 4 components make up the Content Management System, which is the core of the E-commerce system [15]. The following diagram shows how these components fit together:

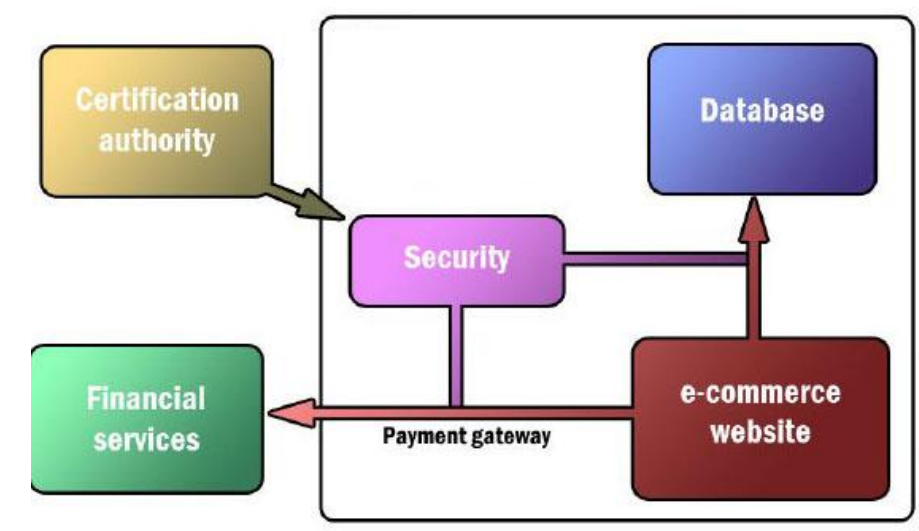

Figure 4.1: Components of an e-commerce content management system

Each of the 4 components of a CMS will be described in more detail in the rest of this section.

\subsubsection{Database}

We are going to use MySQL because it is characterized as a free, fast, reliable open source relational database. It does lack some sophistication and facilities, but it has an active development team and, as it goes from release to release, more capabilities are added. I choose this database because

- Because of its unique storage engine architecture MySQL performance is very high and lightweight.

- Supports large number of embedded applications which makes MySQL very flexible.

- Use of Triggers, Stored procedures and views which allows the developer to give a higher productivity.

- Allows transactions to be rolled back, commit and crash recovery. 
The Design And Implementation of E-Commerce Management System.

\subsubsection{Website}

The website of the e-commerce system is the interface which a user, such as an online shopper, will use to interact with the business. This means that all the information relevant to the user has to be displayed on the website in a comprehensive manner. This information will include product information, order status, shipping and billing addresses, etc. Much research has gone into making e-commerce website interfaces more userfriendly and helpful to the online shopper. Recommender systems [2] are an example of this as they present the online shopper with product recommendations based on the shopper's purchase history. Studies have shown that by doing this, e-commerce businesses can increase their sales volumes.

An e-commerce front-end can be either an application or a website [10]. The former can be written in practically any programming language that can interface with a database (C++, Java, Visual Basic, etc.). The latter can be developed using a web language (PHP, HTML, ASP, etc.).

\subsubsection{SECURITY}

As electronic commerce grows, so does the number of online commercial transactions involving the exchange of sensitive information, such as credit card details and people's personal information [8]. This brings a need for better privacy and security as more and more online shoppers are exposed to hacking and fraud.

A widely used solution to the problem of fraud in e-commerce is certification authorities such as Thawte and VeriSign [11]. These authorities can issue a certificate to an e-commerce business only if it meets certain security standards. This ensures that the two parties involved in an online commercial transaction are really who they claim to be.A widely used solution to the hacker problem is a Secure Socket Layer (or SSL) [12], which allows information exchanged between two parties to be encrypted. This can help prevent any unauthorized third party from intercepting sensitive information.

\subsubsection{PAYMENT GATEWAY}

An online business transaction cannot occur without some sort of payment gateway. A payment gateway allows funds to be transferred electronically from the purchaser to the seller. In the late 90's a non-bank payment service was introduced to electronic commerce: PayPal [14]. Today, this eBay owned service allows consumers to make online credit purchases and even transfer funds to another person (called $\mathrm{C} 2 \mathrm{C}$ or consumerto-consumer transactions).

\subsection{DEVELOPMENT PROCESS}

There are many ecommerce system but most don't have some features like SMS confirmation in Bangladesh based and export date for other software use available on the market.However, if I want to develop online e-commerce system from scratch, one can use a 5-step development process

Step 1: Requirements analysis

Step 2: Sub-system selection

Step 3: Prototype development

Step 4: GUI development

Step 5: Integration and system test



Figure 5: Flow chart of the shopping process 


\section{System Models}

The E-commerce portal will consist of a set of many interacting objects or entities. Using Object Oriented Analysis, the following objects were extracted from the project requirement

\subsubsection{CUSTOMER}

- First name and surname

- Shipping address

- Billing address

- Telephone number

- E-mail address

\subsubsection{ORDER}

- List of Products

- Customer who placed the order

- Total value

- Payment method

- Date created

- Status

\subsection{Business Process Modeling}

\subsubsection{PRODUCT}

- Price

- Product description

- Supplying vendor

- Quantity available

- Category

\subsubsection{IMAGE}

- Image description

- Filename

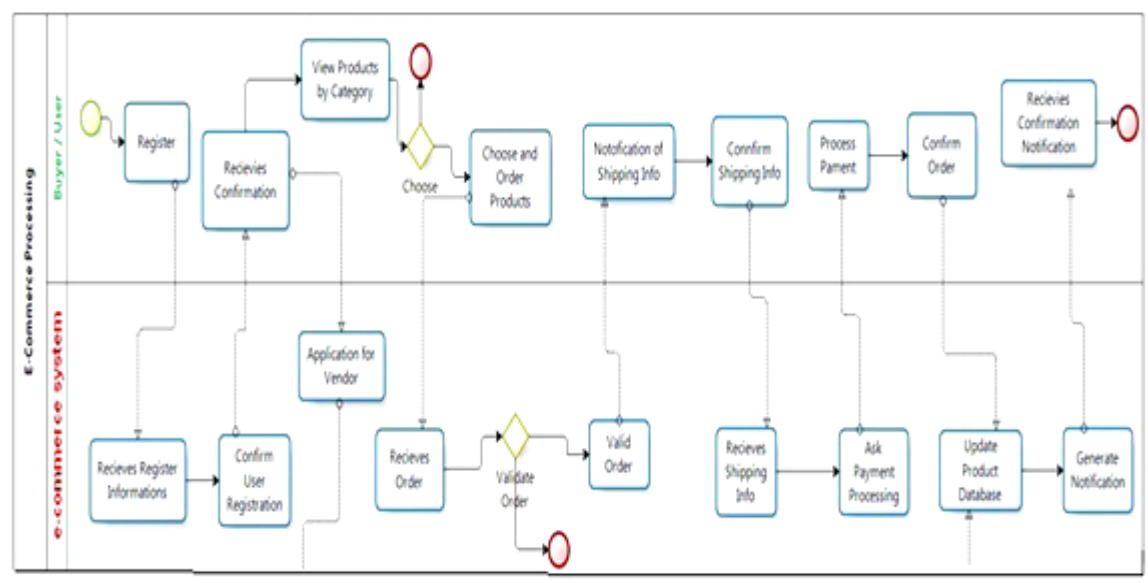

Figure 6.1: Business Process Model

\subsection{Data Flow Diagram}

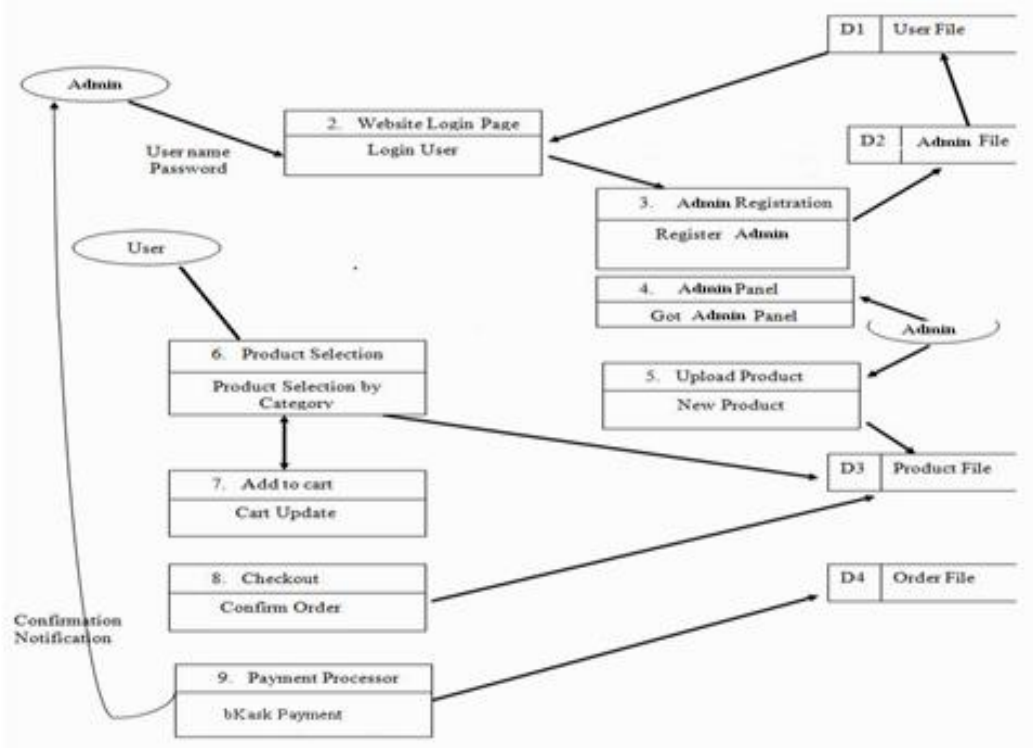

Figure 6.1: Data Flow Diagram 


\subsection{Process Modeling}

\section{Diagram}

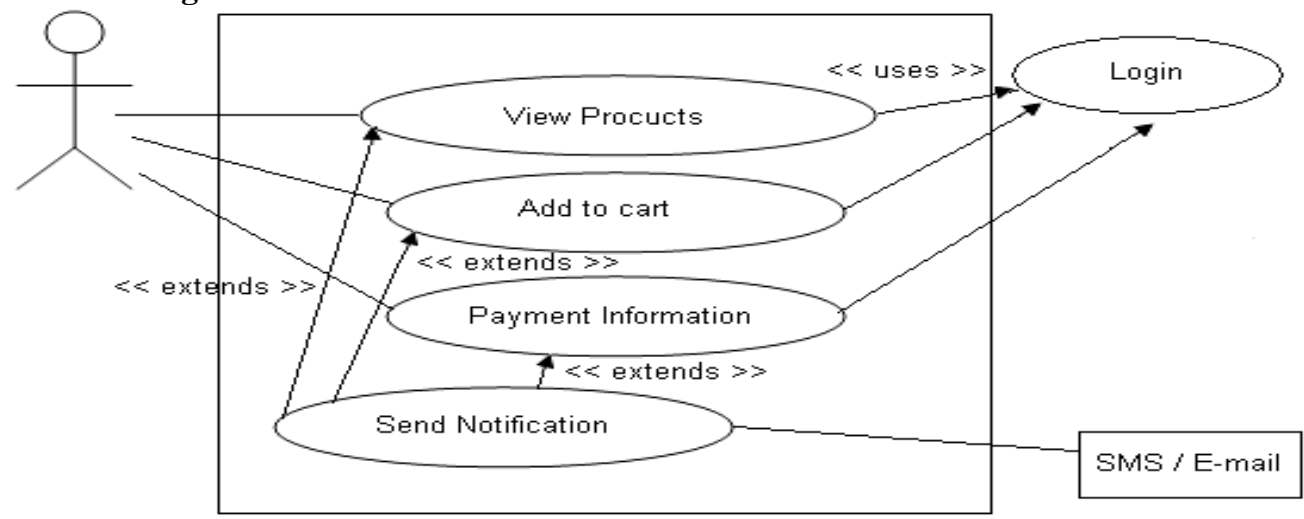

Figure 7.1: Use Case Diagram for buying product

Here the user will come to the shop and view the product information. Then the user will add the product to the cart and finally place the payment information.

\section{Use Case Diagram for Admin}

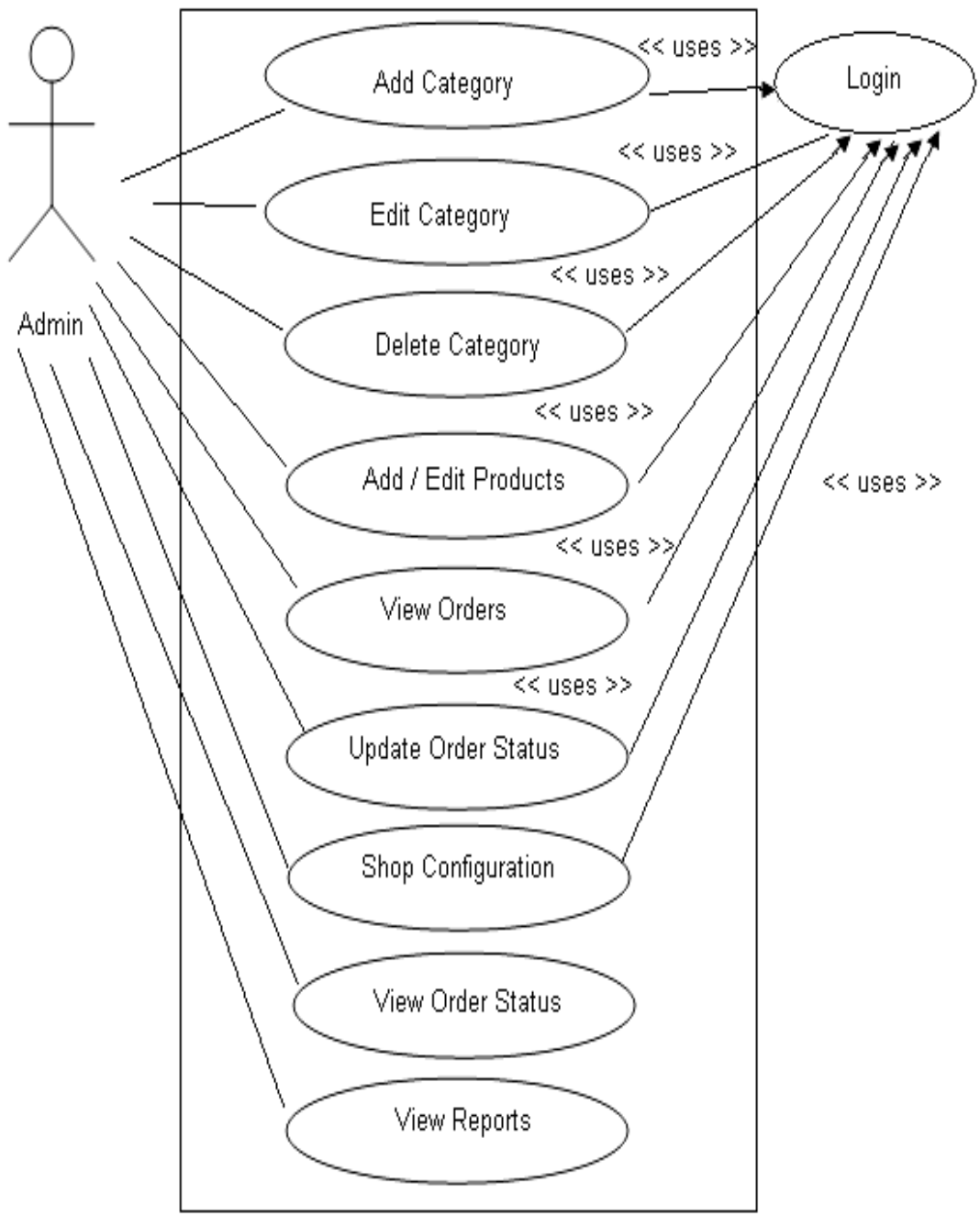

Figure 7.2: Use Case Diagram for Admin 
Admin can add edit categories and products and control the stock. They can check the payment status and update the payment status if required

\subsection{Admins Add product and category}

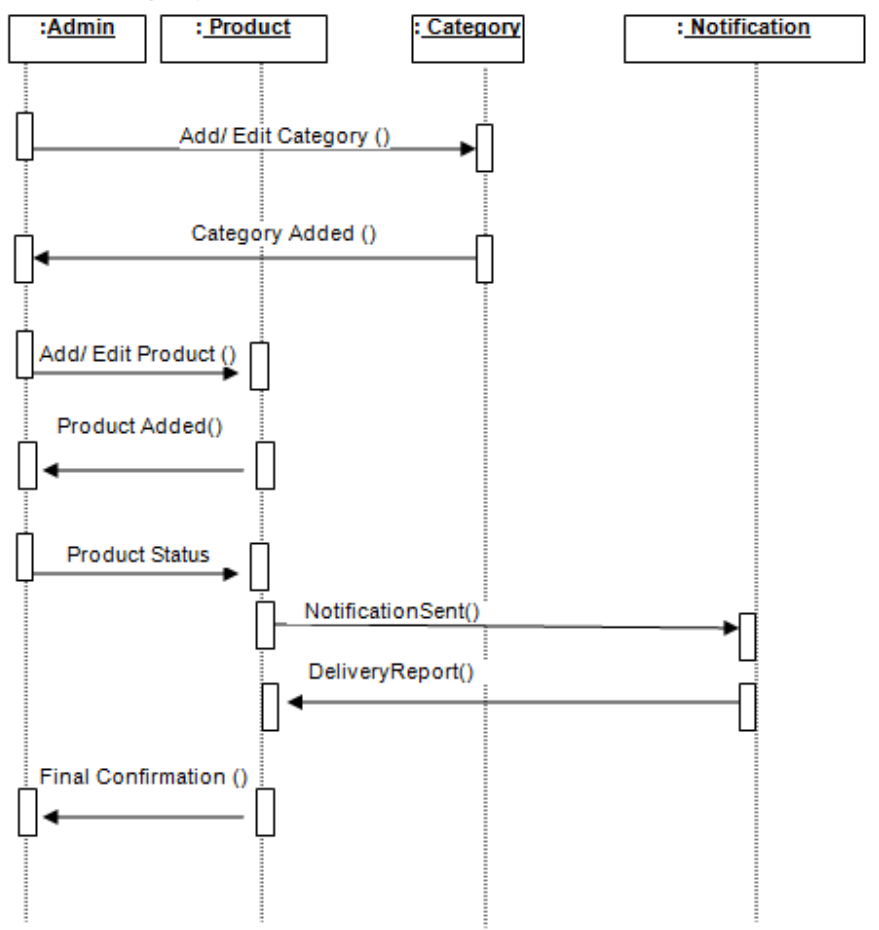

Figure 7.3: Admin's add product and category

\subsubsection{Sequence diagram for product shopping}



Figure 7.4: Sequence diagram for product shopping 


\section{Product Shopping Feature}

\section{Outcomes}

Shopping cart seems to be a web pages containing products this will contain a link "Add to Cart" button; selecting an item for purchase this will add the product and you will be able to see the products quantity are increased and similarly you will be able add multiple products.On the inside, a shopping cart is maintained by PHP Sessions allows you to add and delete products, set up categories, take orders, and sometimes other functionalities such as allowing you to email shipping information to customers etc.On the Shopping Cart Page, the users can modify modifies the quantity of an Item in the Shopping Cart, and then presses the Update button. The system stores the new quantity, and then computes and displays the new cost for that Item.

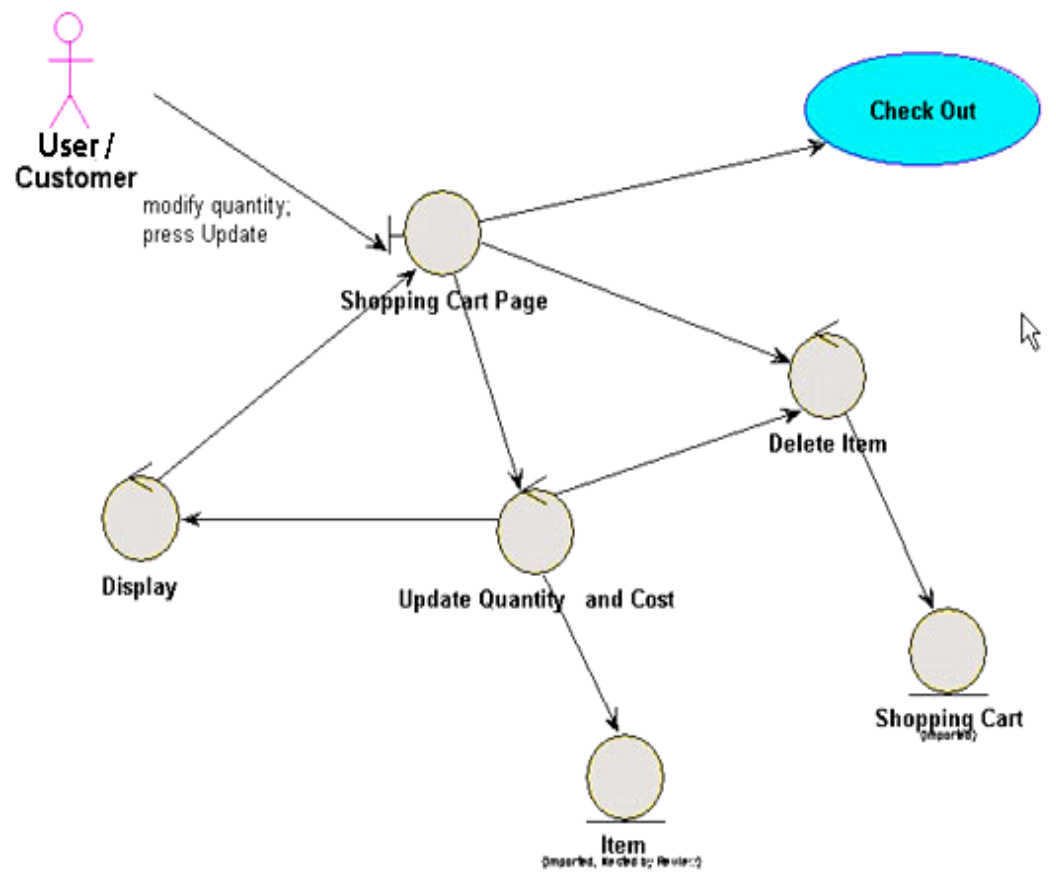

Figure 4.8: Use-case diagram for product shopping

\section{Conclusion}

This paper represents the full development and evaluation process of the e-commerce portal. The aim of the paper was to create an e-commerce portal that allows products buyer's a single one stop marketplace or home to purchase their products. They can also manage their products, payments, currencies etc. The design process involved usability studies into how the e-commerce website should be presented to the users as well as to determine the best design for the customer administration feature and the vendor portal. Using use-cases, website models, and database models, a final design for the system was successfully developed.The implementation process involved setting up a ecommerce System. All the features were developed in PHP, to maintain consistency with the rest of the System and to ensure seamless integration of the new features with the original System. The vendor feature gave customers access to their personal details, their product information

\section{Future Work}

The country needs intermediary institutions to complete transactions. And fortunately, banks, Visa and Master Card operating in Bangladesh, can do it. Bangladesh has the infrastructure to start. Stable internet connection and a law supporting e-commerce are prerequisites. The government needs to make a realistic ecommerce law. It should work in collaboration with the private sector to ensure a stable and reliable internet connection. E-commerce facilitates payment of utility bills, fees etc.

Limited time and resources was the key bindings for this project. While doing the project many important features had been skipped for the simplicity of the project. Below some points showing the future considerations of this project:

- The involvement of local bank credit card transaction can be implemented.

- The entire project can be implemented online to get the realistic feedback from its users. 


\section{References}

[1]. Central Bank of Bangladesh, Bangladesh Bank. <http://www.bangladeshbank.org/econdata/export/exp_rcpt_country_commodity.php> accessed during 2009-2010.

[2]. Schafer, J. B., Konstan, J., and Riedi, J. 1999. Recommender systems in e-commerce. In Proceedings of the 1st ACM Conference on Electronic Commerce (Denver, Colorado, United States, November 03 - 05, 1999). EC '99. ACM Press, New York, NY, 158-166.

[3]. Inernet users in Bangladesh <http://www.internetworldstats.com/asia.htm\#bd>

[4]. Export Promotion Bureau, Bangladesh (EPB), <www.epbbd.com/>, accessed during 2003- 2007.

[5]. Bichler, M., Segev, A., and Zhao, J. L. 1998. Component-based ecommerce: assessment of current practices and future directions. SIGMOD Rec. 27, 4 (Dec. 1998), 7-14

[6]. Bangladesh - Telecoms, Mobile, Broadband and Forecasts $<$ http://telecommarketreserach.com $/ \mathrm{reseach}>7$

[7]. Fingar, P. 2000. Component-based frameworks for e-commerce. Commun. ACM 43, 10 (Oct. 2000), 61-67.

[8]. Portillo, E. and Patel, A. 1999. Design methodology for secure distributed transactions in electronic commerce. Comput. Stand. Interfaces 21, 1 (May. 1999), 5-18.

[9]. Sherif, M., Serhrouchni, A., Gaid, A., Farazmandnia, F. 1998. SET and SSL: Electronic Payments on the Inernet. Third IEEE Symposium on Computers \& Communications (Jun. 1998). 153-155.

[10]. Database Systems: Design, Implementation and Management by Peter Rob

[11]. Andres, G. 2003. PayPal and eBay: The legal implications of the C2C electronic commerce model. 18th BILETA Conference: Controlling Information in the Online Environment (Apr. 2003).

[12]. Server-side HTML embedded scripting language. It provides web developers with a full suite of tools for building dynamic websites. 〈www.php.net>

[13]. Ghosh, A. K. and Swaminatha, T. M. 2001. Software security and privacy risks in mobile e-commerce. Commun. ACM 44, 2 (Feb. 2001), 51-57. 